\title{
Searching for the most distant blazars with the Fermi Gamma-ray Space Telescope
}

\author{
Yoshiyuki Inoue $^{a}$, Susumu Inoue ${ }^{b}$ Masakazu A. R. Kobayashi, ${ }^{c}$ Tomonori Totani $^{a}$, \\ Jun Kataoka ${ }^{d}$, \& Rie Sato ${ }^{e}$ \\ ${ }^{a}$ Department of Astronomy, Kyoto University, Kitashirakawa Oiwake-cho, Sakyo-ku, Kyoto \\ 606-8502, Japan \\ ${ }^{b}$ Department of Physics, Kyoto University, Kitashirakawa Oiwake-cho, Sakyo-ku, Kyoto \\ 606-8502, Japan \\ ${ }^{c}$ Optical and Infrared Astronomy Division, National Astronomical Observatory of Japan, Mitaka, \\ Tokyo 181-8588, JAPAN \\ ${ }^{d}$ Waseda University, 1-104 Totsukamachi, Shinjuku-ku, Tokyo 169-8050, Japan \\ ${ }^{e}$ Department of High Energy Astrophysics, Institute of Space and Astronautical Science (ISAS), \\ Japan Aerospace Exploration Agency (JAXA), 3-1-1 Yoshinodai, Sagamihara, 229-8510, Japan \\ E-mail: yinouedkusastro.kyoto-u.ac.jp
}

\begin{abstract}
We investigate the prospects for discovering blazars at very high-redshifts $(z>3-6)$ with the Fermi Gamma-Ray Space Telescope (Fermi), employing a model for the evolving gamma-ray luminosity function (GLF) of the blazar population. Our previous GLF model is used as a basis, which features luminosity-dependent density evolution implied from X-ray data on active galactic nuclei, as well as the blazar sequence paradigm for their spectral energy distribution, and which is consistent with EGRET and current Fermi observations of blazars. Here we augment the highredshift evolution of this model by utilizing the luminosity function of quasars from the Sloan Digital Sky Survey (SDSS), which is well-constrained up to $z \sim 5$. We find that Fermi may discover a few blazars up to $z \sim 6$ in the entire sky during its 5 -year survey. We also discuss how such high-redshift blazar candidates may be efficiently selected in future Fermi data. We further discuss that the GeV gamma-ray spectra of such high-redshift blazars would be a probe of the early cosmic star formation history.
\end{abstract}

25th Texas Symposium on Relativistic Astrophysics - TEXAS 2010

December 06-10, 2010

Heidelberg, Germany 


\section{Introduction}

High-energy gamma-ray astronomy has progressed drastically after the launch of the Fermi Gamma-ray Space Telescope (Fermi). Its Large Area Telescope (LAT) is currently observing the entire gamma-ray sky in the $0.02-300 \mathrm{GeV}$ range. The majority of extragalactic sources detected by Fermi are blazars, a subclass of active galactic nuclei (AGNs) dominated by broadband, nonthermal emission arising from relativistic jets oriented close to our line of sight. The Fermi 11 month catalog reports the detection of $\sim 600$ blazars up to $z \sim 3$ [1].

Before the Fermi era, the Energetic Gamma-Ray Experiment Telescope (EGRET) detected $\sim 50$ blazars in total up to $z \sim 3$ [2]. Since Fermi has an order of magnitude better sensitivity and position accuracy compared to EGRET, it is naturally expected that Fermi will see much deeper into the universe in gamma-rays.

The purpose of this paper is to discuss expectations for the highest redshift blazars that Fermi may discover. This requires reasonable knowledge of their gamma-ray luminosity function (GLF). The blazar GLF has been discussed from different perspectives in many papers so far (see e.g. [3]). Inoue \& Totani (2009; hereinafter IT09) [3] and Inoue, Totani \& Mori (2010; hereinafter ITM09) [4] have recently developed a new model of the blazar GLF that accounts for the blazar spectral sequence as well as luminosity-dependent density evolution, and which is consistent with the EGRET and current Fermi data 44,5]. However, the ITM10 GLF is uncertain for $z>3$, because the current observed number of X-ray AGNs and gamma-ray blazars above $z \sim 3$ is insufficient to strongly constrain the model. On the other hand, the optical luminosity function (OLF) of AGNs has been well constrained up to $z \sim 5$ [6]. Therefore, here we consider a modified blazar GLF by combining the AGN XLF with the evolutionary constraints from the AGN OLF data.

Throughout this proceeding, we adopt the standard cosmological parameters of $\left(h, \Omega_{M}, \Omega_{\Lambda}\right)=$ $(0.7,0.3,0.7)$.

\section{High Redshift Evolution of the Blazar Gamma-ray Luminosity Function}

\subsection{Blazar Gamma-ray Luminosity Function}

IT09 recently developed a model for the blazar GLF featuring the so-called luminosity-dependent density evolution (LDDE), based on the latest determination of the AGN XLF [7, 8]. Another novel aspect of IT09 is accounting for the blazar sequence of their spectral energy distribution (SED). The blazar sequence refers to the observed trend whereby the two characteristic frequencies at where the blazar SED peaks systematically decrease as the bolometric luminosity increases [9 10]. The key parameters in the GLF model have been carefully determined to match the observed flux and redshift distribution of EGRET blazars by a likelihood analysis. Although the blazar sequence SED in IT09 was observationally constrained only up to the EGRET band of $30 \mathrm{GeV}$, this was extended to include the $\mathrm{TeV}$ band in ITM10, using published $\mathrm{TeV}$ blazar data. In this paper, we use the ITM10 GLF model as a baseline model.

\subsection{Constraints on the High Redshift Evolution of Blazars}

The ITM10 GLF model is based on data from EGRET blazars [2] and X-ray AGNs [7, 8], the highest redshifts for both samples being $z \sim 3$. To address the evolution at $z \geq 3$, additional obser- 


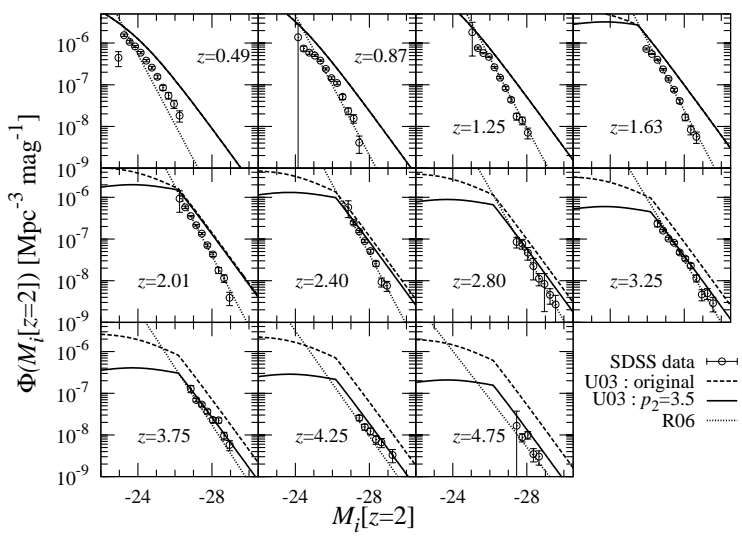

Figure 1: $M_{i}[z=2]$ luminosity function at each redshift as indicated in each panel. Circles indicate SDSS quasar OLF data [6. Dashed, solid, and dotted curves show the U03 original XLF $\left(p_{2}=1.5\right)$, modified U03 XLF ( $\left.p_{2}=3.5\right)$, and R06 OLF, respectively.

vational constraints are necessary. Optical surveys such as the Sloan Digital Sky Survey (SDSS) has successfully identified quasars up to $z=6.43$ [11], and the AGN OLF is well determined up to $z \sim 5$ (Richards et al 2006; hereinafter R06). Utilizing the R06 OLF, below we modify the high redshift evolution of our previous best-fit blazar GLF based on the AGN XLF of Ueda et al. (2003 [7]; hereafter U03). The AGN XLF and OLF are merged following the procedures in $\$ 5.4$ of [12] and $\S 3,4$ in U03.

The power-law index $p_{2}$ in the U03 XLF characterizes the density evolution as a function of $z$ at high redshift. Although $p_{2}=1.5$ was used in ITM10, here we modify this to $p_{2}=3.5$ in order to be consistent with the high-redshift R06 OLF data. Since $p_{2}$ does not affect the low-redshift evolution, the XLF is not significantly altered below $z \sim 3$. Fig. 1] shows the AGN OLF at each redshift as in terms of $M_{i}[z=2]$ in comparison with the R06 OLF data. With this AGN XLF, we reconstruct the blazar GLF with the ITM10 blazar sequence SED model, following the procedures of IT09.

The key parameters of our new blazar GLF model are $\left(q, \gamma_{1}, \kappa\right)=\left(4.42,1.07,1.92 \times 10^{-6}\right)$, where $q$, the ratio between the bolometric jet luminosity and nuclear X-ray luminosity, $\gamma_{1}$, the faintend slope index of the GLF, and $\kappa$, a normalization factor for the GLF (see Section. 3 of IT09 for details). Base on this model, we make predictions for the high-redshift blazars that Fermi may discover.

\section{Predictions for high-redshift Fermi blazars}

\subsection{Expected Redshift Distribution of Fermi Blazars}

Fig. 2] shows the expected cumulative redshift distribution of Fermi blazars above $100 \mathrm{MeV}$ in the entire sky for a $\sim 5$-year survey flux sensitivity limit of $F(>100 \mathrm{MeV})=1 \times 10^{-9}$ photons $/ \mathrm{cm}^{2} / \mathrm{s}$. Neglecting high-redshift optical constraints, Fermi may be able to detect blazars up to $z \sim 10$. Taking into account their high-redshift evolution implied from the AGN OLF, we expect that Fermi will find blazars up to $z \sim 6$ with the $\sim 5$-year survey sensitivity. 


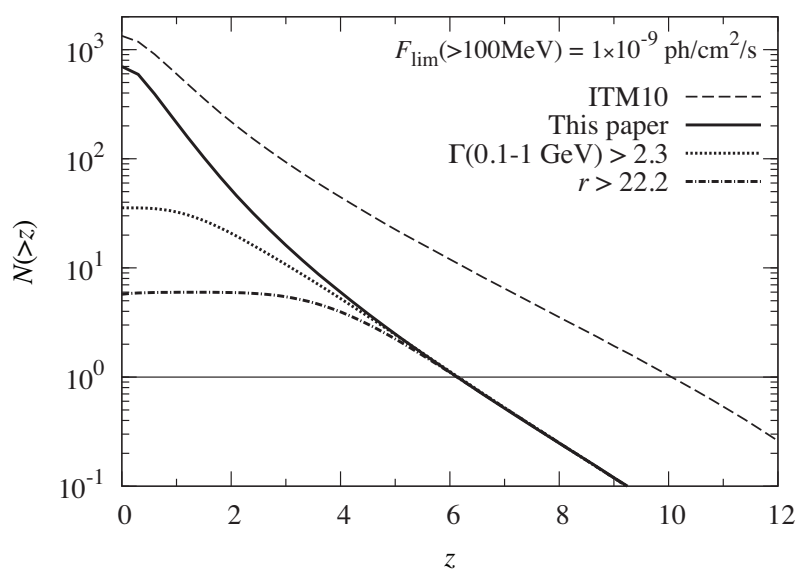

Figure 2: Expected cumulative redshift distribution of blazars detectable by Fermi above $100 \mathrm{MeV}$ in the entire sky for a flux sensitivity limit $F(>100 \mathrm{MeV})=1 \times 10^{-9}$ photons $/ \mathrm{cm}^{2} / \mathrm{s}$. Solid and dashed curves correspond to whether or not AGN OLF constraints are taken into account. The dotted curve is for $\Gamma>2.3$, and the dot-dashed curve is for $\Gamma>2.3$ together with $r>22.2$

\subsection{Methods for selecting high-redshift blazar candidates}

It is expected that Fermi will find more than 1,000 blazars [3]. Although $\sim 70 \%$ of the Fermi blazars have confirmed redshifts, the other $\sim 30 \%$ still do not [1]. This implies that the highest redshift blazars must be searched for among a large number of sources and distinguished from numerous unrelated ones. The following methods for their observational selection should be effective in searching for high-redshift blazars.

First, we select the sources whose fluxes are close to the Fermi sensitivity limit, as the highredshift blazars are naturally expected to be faint. Furthermore, it should be useful to look those showing time variability, a characteristic trait of blazars.

Second, we choose the sources whose $0.1-1 \mathrm{GeV}$ photon indices $\Gamma>2.3$ to pick out luminous blazars with gamma-ray luminosities $L_{\gamma}>10^{47} \mathrm{erg} / \mathrm{s}$ at $100 \mathrm{MeV}$, since high-redshift objects that are detectable should be luminous, and their spectral indices are expected to be soft according to the blazar sequence [9, 10.

Third, we identify the radio counterpart using deep radio survey catalogs, which should give us tighter constraints on the source location and identification. The radio fluxes of blazars at $z>6$ expected from the blazar sequence are $\sim 20 \mathrm{mJy}$. These are below the limiting sensitivity of radioloud galaxy catalogs used for the current Fermi catalog. More useful for our purposes are the Faint Images of the Radio Sky at Twenty centimeters survey (FIRST) [13] and the NRAO VLA Sky Survey (NVSS) [14 catalogs, which go down to $1 \mathrm{mJy}$ at $1.4 \mathrm{GHz}$, below the expected 1.4 $\mathrm{GHz}$ radio flux for blazars at $z>6$. Within the Fermi localization uncertainty of $\sim 10^{\prime}$ at Galactic latitudes $|b|>10^{\circ}$, the expected radio source count is $\sim 0.20$ blazars. Therefore, identifying the radio counterpart should be essential.

Fourth, we select objects that are not detected in optical surveys such as the SDSS, in view of the unavoidable attenuation due to intergalactic HI for high-redshift sources. Since FIRST covers the SDSS survey area, a quarter of the entire sky, sources detected by the SDSS can be searched 
for their radio counterparts. Here we set the optical selection criterion to be $r>22.2$ in AB magnitude, the $r$-band limiting magnitude of SDSS. We caution that the SDSS sensitivities in the $i$ and $z$ band would be insufficient for detecting blazars at $z>3$ even in the absence of intergalactic absorption. Thus, the SDSS data by itself cannot be used for identifying high-redshift blazars, but will nevertheless be valuable for rejecting low-redshift contaminants.

The dotted and dot-dashed curves in Fig. 2 2 show cases where the above selection criteria have been imposed for the gamma-ray photon index and the optical magnitude. The expected total number of Fermi blazars would decrease to $\sim 60$ sources, of which $\sim 38 \%$ is expected to lie at $z>5$, so this procedure should be effective in narrowing down high-redshift blazar candidates, albeit with some remaining low-redshift contamination. Finally, deeper optical and infrared follow-up observations of the candidate objects are warranted to accurately identify their respective counterparts and spectroscopically measure their actual redshifts. Note that for blazars in the southern sky, surveys such as that being undertaken by the South Pole Telescope at 1.4 and $2.0 \mathrm{~mm}$ with milli-Jansky sensitivities [15], as well as the VST ATLAS and VISTA surveys in the optical and near-infrared, should prove to be likewise valuable for their selection.

\section{Probing the Cosmic Star Formation History at $z>6$}

It is well known that high energy $\gamma$-rays $(\gtrsim 20 \mathrm{GeV})$ propagating the universe are absorbed by the interaction with the extragalactic background light (EBL), also called as cosmic optical and infrared background [16-18]. Here GeV photons from high-redshift sources are also expected to suffer intergalactic absorption due to electron-positron pair production interactions but with the high redshift cosmic UV background radiation [18-20].

By using the semi-analytical galaxy formation model [21], we have estimated the gamma-ray optical depth from $z=20$ to $z=0$ [22]. In Figure. 3, we show the expected gamma-ray optical depth comparing with previous works [16, 20]. The resulting absorption features in the spectra of such sources could therefore be a key probe of the poorly-understood UV background radiation and provide valuable insight into the cosmic dark ages. Discovering high-redshift gamma-ray blazars or gamma-ray bursts will thus offer a new probe of the formation histories of early stars and galaxies.

\section{Summary}

We studied the prospects for detecting the highest-redshift blazars with Fermi, and how we may select such blazars from the numerous other expected Fermi sources. The high-redshift evolution of the blazar GLF was constrained from the observed AGN OLF, which is well determined up to $z \sim 5$. Thus we found that Fermi may discover some blazars up to $z \sim 6$ down to the $\sim 5$-year survey flux sensitivity limit of $F(>100 \mathrm{MeV})=1 \times 10^{-9}$ photons $/ \mathrm{cm}^{2} / \mathrm{s}$.

High-redshift blazar candidates may be selected through the criteria that the source (1) is faint and shows time variability, (2) has a soft spectrum with $\Gamma>2.3$, corresponding to luminous blazars in the blazar sequence, (3) has a radio counterpart in deep survey catalogs such as FIRST, and (4) are not detected in the SDSS, which should remove low-redshift contamination. Then such sources must be followed up by optical and infrared telescopes. 


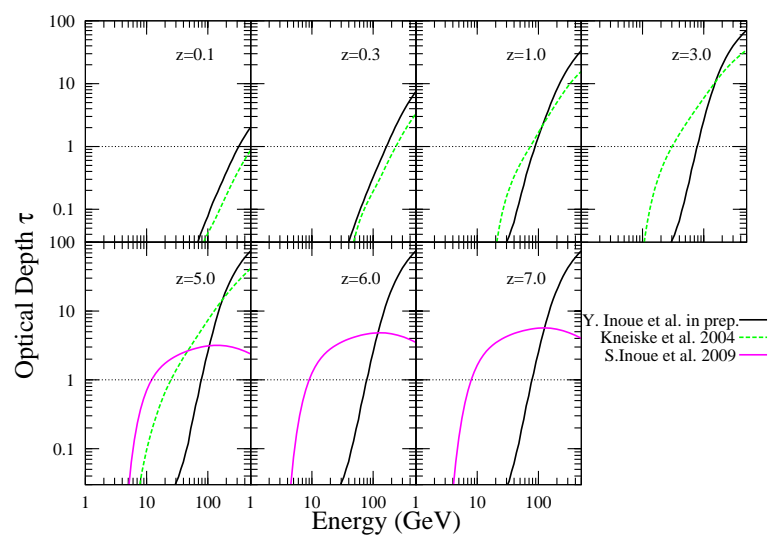

Figure 3: Optical depth of intergalactic absorption of high-energy gamma-rays for various source redshifts, as indicated in each panel. The black, green, and magenta curves correspond to the models of Y. Inoue et al. in prep. [22], Kneiske et al. (2004) [16], and S. Inoue et al. (2009) [20], respectively. The dotted line marks the level of the optical depth, $\tau=1$.

\section{acknowledgments}

This work was supported in part by the Global COE Program "The Next Generation of Physics, Spun from Universality and Emergence" at Kyoto University from the Ministry of Education, Culture, Sports, Science and Technology (MEXT) of Japan. YI acknowledges support by the Research Fellowship of the Japan Society for the Promotion of Science (JSPS), and SI acknowledges support by the Grant-in-Aid No.22540278 from MEXT of Japan.

\section{References}

[1] A. A. Abdo, et al., The First Catalog of Active Galactic Nuclei Detected by the Fermi Large Area Telescope, Astrophysical Journal, 715, 429 (2010) [arXiv: 1002 .2280]

[2] R. C. Hartman, et al., The Third EGRET Catalog of High-Energy Gamma-Ray Sources, Astrophysical Journal, Supplement, 123, 79 (1999)

[3] Y. Inoue, \& T. Totani, The Blazar Sequence and the Cosmic Gamma-ray Background Radiation in the Fermi Era, Astrophysical Journal, 702, 523 (2009) [arXiv: 0810 . 3580]

[4] Y. Inoue, T. Totani, \& M. Mori, Prospects for a Very High-Energy Blazar Survey by the Next-Generation Cherenkov Telescopes, PASJ, 62, 1005 (2010) [arXiv: 1002. 4782]

[5] Y. Inoue, S. Inoue, M. A. R. Kobayashi, T. Totani, J. Kataoka, \& R. Sato, Searching for the most distant blazars with the Fermi Gamma-ray Space Telescope, Monthly Notices of the Royal Astronomical Society, 411, 464 (2011) [arXiv: 1007 .4379]

[6] G. T. Richards, et al., The Sloan Digital Sky Survey Quasar Survey: Quasar Luminosity Function from Data Release 3, Astronomical Journal, 131, 2766 (2006) [arXiv: astro-ph/ 0601434 ]

[7] Y. Ueda, M. Akiyama, K. Ohta, \& T. Miyaji, Cosmological Evolution of the Hard X-Ray Active Galactic Nucleus Luminosity Function and the Origin of the Hard X-Ray Background, Astrophysical Journal, 598, 886 (2003) [arXiv: astro-ph/0308140] 
[8] G. Hasinger, T. Miyaji, \& M. Schmidt, Luminosity-dependent evolution of soft X-ray selected AGN. New Chandra and XMM-Newton surveys, A\&A, 441, 417 (2005) [arXiv: astro-ph / 0506118 ]

[9] G. Fossati, L. Maraschi, A. Celotti, A. Comastri, \& G. Ghisellini, A unifying view of the spectral energy distributions of blazars, Monthly Notices of the Royal Astronomical Society, 299, 433 (1998) [arXiv:astro-ph/9804103]

[10] H. Kubo, T. Takahashi, G. Madejski, M. Tashiro, F. Makino, S. Inoue, \& F. Takahara, ASCA Observations of Blazars and Multiband Analysis, Astrophysical Journal, 504, 693 (1998) [arXiv:astro-ph/9804031]

[11] X. Fan, et al., A Survey of z>5.7 Quasars in the Sloan Digital Sky Survey. II. Discovery of Three Additional Quasars at z>6, Astronomical Journal, 125, 1649 (2003)

[arXiv:astro-ph/0301135]

[12] G. T. Richards, et al., The 2dF-SDSS LRG and QSO (2SLAQ) Survey: the $z<2.1$ quasar luminosity function from 5645 quasars to $g=21.85$, Monthly Notices of the Royal Astronomical Society, 360, 839 (2005) [arXiv:astro-ph/0504300]

[13] R. L. White, R. H. Becker, D. J. Helfand, \& M. D. Gregg, A Catalog of 1.4 GHz Radio Sources from the FIRST Survey, Astrophysical Journal, 475, 479 (1997)

[14] J. J. Condon, W. D. Cotton, E. W. Greisen, Q. F. Yin, R. A. Perley, G. B. Taylor, \& J. J. Broderick, The NRAO VLA Sky Survey, Astronomical Journal, 115, 1693 (1998)

[15] J. D. Vieira, et al., Extragalactic Millimeter-wave Sources in South Pole Telescope Survey Data: Source Counts, Catalog, and Statistics for an 87 Square-degree Field, Astrophysical Journal, 719, 763 (2010) [arXiv:0912.2338]

[16] T. M. Kneiske, T. Bretz, K. Mannheim, \& D. H. Hartmann, Implications of cosmological gamma-ray absorption. II. Modification of gamma-ray spectra, A\&A, 413, 807 (2004) [arXiv:astro-ph/0309141]

[17] A. Franceschini, G. Rodighiero, \& M. Vaccari, Extragalactic optical-infrared background radiation, its time evolution and the cosmic photon-photon opacity, $A \& A, 487,837$ (2008)

[arXiv:0805.1841]

[18] R. C. Gilmore, P. Madau, J. R. Primack, R. S. Somerville, \& F. Haardt, GeV gamma-ray attenuation and the high-redshift UV background, Monthly Notices of the Royal Astronomical Society, 399, 1694 (2009) [arXiv:0905.1144]

[19] S. P. Oh, Probing High-Redshift Radiation Fields with Gamma-Ray Absorption, Astrophysical Journal, 553, 25 (2001) [arXiv:astro-ph/ 0005263 ]

[20] S. Inoue, R. Salvaterra, T. R. Choudhury, A. Ferrara, B. Ciardi, \& R. Schneider, Probing intergalactic radiation fields during cosmic reionization through gamma-ray absorption, Monthly Notices of the Royal Astronomical Society, 404, 1938 (2010) [arXiv: 0906.2495 ]

[21] M. A. R. Kobayashi, T. Totani, \& M. Nagashima, Ly $\boldsymbol{\alpha}$ Emitters in Hierarchical Galaxy Formation. II. Ultraviolet Continuum Luminosity Function and Equivalent Width Distribution, Astrophysical Journal, 708, 1119 (2010) [arXiv:0902 .2882]

[22] Y. Inoue, et al. in preparation. 